\title{
Membrane Fluidity of Polymorphonuclear Leukocytes from Children with Primary Ciliary Dyskinesia
}

\author{
AHMAD KANTAR, NICOLA OGGIANO, PIER LUIGI GIORGI, AND ROSAMARIA FIORINI \\ Department of Pediatrics [A.K., N.O., P.L.G.] and Institute of Biochemistry [R.F.], University of Ancona,
} Ancona, Italy

\begin{abstract}
Plasma membrane fluidity and heterogeneity of polymorphonuclear leukocytes (PMN) were investigated in seven children with primary ciliary dyskinesia (PCD) and 17 healthy controls. Membrane fluidity and heterogeneity were studied by measuring the steady state fluorescence anisotropy and fluorescence decay of 1-(4-trimethylammoniumphenyl)-6-phenyl-1,3,5-hexatriene (TMADPH) incorporated into PMN plasma membrane. Our results show an increase in membrane fluidity at the surface level of PMN from patients with PCD. Distribution analysis of TMA-DPH lifetime values indicate an increase in membrane heterogeneity in subjects with PCD. The observed changes in the physicochemical properties of the membrane could lead to alterations in the function of PMN from children with PCD. (Pediatr Res 34: 725-728, 1993)
\end{abstract}

\section{Abbreviations}

PMN, polymorphonuclear leukocyte PCD, primary ciliary dyskinesia

TMA-DPH, 1-(4-trimethylammoniumphenyl)-6-phenyl1,3,5-hexatriene

FMLP, N-formyl-methionyl-leucyl-phenylalanine

$r_{s}$, steady state fluorescence anisotropy

PMA, phorbol myristate acetate

PMN from subjects with PCD can have an abnormal locomotory system. Defects in orientation (1,2), migration (1-3) and chemotaxis (3-5) have been reported. All these activities are the result of complex events mediated by the plasma membrane and cytoskeleton of PMN. Specific and dynamic interactions of plasma membrane components are essential for an adequate membrane function (6). Membrane fluidity has an important role in modulating cell functions, affecting the conformation of membrane proteins and the exposure and diffusion of membrane components. Fluidity is a complex physicochemical feature that depends upon mobility and order of membrane constituents (6, 7). Changes in composition and molecular organization are the principal determinants of alterations of membrane fluidity observed in many human diseases $(8,9)$.

In this study, we have evaluated plasma membrane fluidity of PMN obtained from children with PCD by measuring $r_{s}$ and fluorescence decay of a probe incorporated into the plasma membranes. As a fluorescent probe, we have used TMA-DPH, which is incorporated at the lipid-water interface region bilayer.

Received February 9, 1993; accepted July 22, 1993.

Correspondence: Dr. A. Kantar, Department of Pediatrics, University of Ancona, via Corridoni 11, 1-60123 Ancona, Italy.

\section{MATERIALS AND METHODS}

Subjects. Seven children with PCD, five females and two males, with an age range between 1 mo and 9.4 y (mean $6 \pm 3.2$ $y$ ), were included in this study after appropriate informed consent had been obtained. Virtually all these children had a clinical history of upper and lower respiratory tract infections with onset in early childhood characterized by productive cough, rhinorrhea, severe otitis, chronic sinusitis, and recurrent bronchitis or pneumonia. Kartagener's syndrome (situs inversus, chronic sinusitis, and bronchiectasis) was observed in one female patient. Chest radiograph abnormalities present at the time of diagnosis included hyperinflation (six patients), situs inversus (two patients), and/or segmental bronchiectasis (three patients). In all subjects, the diagnosis of PCD was established by studying ciliary motion and ultrastructure. Nasal and bronchial brushings were obtained from each subject and handled for ciliary motion analysis and ultrastructural studies as previously described (10, 11). Analysis of ciliary motion examined by a light microscope equipped with Nomarsky differential interference contrast set as previously described (11) demonstrated a total absence of ciliary motion in three subjects and an uncoordinated and asyncrhonous motion with a reduced beat frequency of $2-6 \mathrm{~Hz}$ (normal range $12 \pm 2.4 \mathrm{~Hz}$ ) in four subjects. Ultrastructural studies, performed as previously described $(10,11)$, revealed microtubular transposition defects, radial spokes defects, and the total or partial lack of dynein arms (inner, outer, or both). Once diagnosis of PCD was confirmed, patients were treated with chest physiotherapy and intermittent courses of systemic antibiotic.

The control group consisted of 17 healthy children, 12 females and five males, between 6 mo and 10.2 y old (mean $6.4 \pm 3.0$ y). None of the children in the control group had a family or personal history of chronic respiratory disease or immunologic disorders.

All subjects were without signs of acute infection and had not suffered from acute infection in the previous $6 \mathrm{wk}$. In the case of the PCD group, patients had completed a course of systemic antibiotic therapy 6 wk earlier.

After overnight fasting, $13 \mathrm{~mL}$ of heparinized blood were obtained for isolation of PMN. Simultaneously, we measured erythrocyte sedimentation rate, differential white blood count in whole blood, IgM, IgA, IgG, complement factors $C_{3}$ and $C_{4}$, and plasma cholesterol levels as previously described (12).

Isolation of PMN. PMN were isolated using a Mono-Poly Resolving Medium (ICN Biomedicals, Milan, Italy) as previously described (13). Cells were resuspended in Krebs-Ringer phosphate solution supplemented with $5 \mathrm{mM}$ glucose.

Chemiluminescence measurements. Luminol-amplified chemiluminescence was measured in the Autolumat LB 953 (Berthold Co., Wilbad, Germany) and PMN were activated by addition of PMA $10^{-6}$ M (Sigma Chemical Co., St. Louis, MO). 
Chemiluminescence measurements were followed for the next $60 \mathrm{~min}$ as previously described (14).

Chemotaxis assay. Chemotaxis was tested by a modified Boyden technique as described by Governa et al. (15) with the use of blind-well chambers and 5- $\mu \mathrm{m}$ pore cellulose acetate filters (Millipore Corp., Bedford, MA). Zymosan-activated serum, prepared as described by Metcalf et al. (16), was used as a chemoattractant. Zymosan was obtained from Sigma Chemical Co. Tissue culture medium 199 (ICN Biomedicals) was used to determine random motility. After incubation of cells for $3 \mathrm{~h}$ at $37^{\circ} \mathrm{C}$, the filters were removed and stained. The number of cells that completely migrated through the filter was counted within 10 microscopic fields $(400 \times)$ using a $5 \times 5-\mathrm{mm}$ photographic reticle, and the chemotactic index was calculated $(15,16)$. Samples were tested in duplicate.

Fluorescence measurements. The $\mathrm{r}_{\mathrm{s}}$ measurements were performed at $37^{\circ} \mathrm{C}$ with a Perkin Elmer Spectrofluorimeter MPF66, equipped with a Perkin Elmer 7300 Personal Computer for data acquisition and elaboration (Perkin Elmer Corp., Norwalk, CT), using TMA-DPH (Molecular Probes Inc., Eugene, OR) as a hydrophobic fluorescent probe at a final concentration of $10^{-6}$ $M$ as previously described (17). The computer program calculated fluorescence anisotropy by using the expression $\left(I_{\|}-I_{\perp} X\right.$ g)/( $\left.I_{\|}+2 I_{\perp} \times g\right)$, where $g$ is an instrumental correction factor and $I_{\|}$and $I_{\perp}$ are the emission intensities with the polarizers parallel and perpendicular respectively to the direction of the polarized exciting light. The optimal cell concentration for fluorescence measurements has been determined as previously described (17). After incubation with TMA-DPH for $5 \mathrm{~min}$, the basal $r_{s}$ was measured for $5 \mathrm{~min}$. PMN were later activated by the addition of FMLP at a final concentration of $10^{-6} \mathrm{M}$ and $r_{s}$ measurements were followed for the next $15 \mathrm{~min}$ as previously described (18).

Fluorescence lifetime measurements were performed as previously described (19) with a multifrequency phase fluorometer. The instrument was equipped with an ADC interface (ISS Inc., Champaign, IL) for data collection and analysis; the excitation wavelength was set at $325 \mathrm{~nm}$ (UV line of a helium/cadmium laser, model 42240 NB, LiCONix, Santa Clara, CA). The range of modulation frequencies used for TMA-DPH was 7-130 MHz. Data were accumulated at each modulation frequency until the $\mathrm{SD}$ of the phase and modulation values were below $0.1^{\circ}$ and 0.002 , respectively. The fluorescence was measured through a long-pass filter (type RG 370 from Janos Technology, Townshend, VT), which showed negligible luminescence. The experimental data were analyzed by a model that assumes a continuous distribution of lifetime values characterized by Lorentzian shape centered at a time $C$ and having a width $W$ as previously described $(20,21)$. For this analysis, the program minimizes the reduced $\chi^{2}$ defined by an equation reported elsewhere (22). The temperature of the samples was maintained at $37^{\circ} \mathrm{C}$ with an external bath circulator.

Statistical method. The nonparametric Mann-Whitney U test was used to determine the significance of differences between the PCD group and the control group. To determine the significance of the data obtained in each group, we used the $t$ test.

\section{RESULTS}

Erythrocyte sedimentation rate, white blood count, serum level of immunoglobulins, $C_{3}, C_{4}$, and plasma cholesterol values from the PCD and control groups were not significantly different $(p>0.5)$.

Luminol-amplified chemiluminescence has been used to verify that in isolated PMN the superoxide-generating oxidase system is dormant under basal conditions and can be activated by PMA. All samples used in this study demonstrated an activatable NADPH-oxidase system (data not shown). Peak activity was noted within 15-20 min of the addition of PMA and there was no significant difference in the peak value of PMA-stimulated chemiluminescence between the two groups $(p>0.5)$.

Chemotactic index of PMN from subjects with PCD ranged between 86 and 110 (mean $94.5 \pm 8.6$ ), whereas in the control group values obtained ranged between 108 and 152 (mean 122 \pm 12.4 ).

The background fluorescence of PMN was checked before each measurement and was less than $0.1 \%$ of the fluorescence when TMA-DPH was added. In basal conditions, without stimulation, the $r_{s}$ of TMA-DPH incorporated in plasma membrane of PMN from the PCD and control groups was stable and did not show significant changes $(p>0.5)$ for 30 min after addition of TMA-DPH. The TMA-DPH $r_{s}$ values of unstimulated PMN from the PCD and control groups are reported in Table 1. The basal values for PMN from subjects with PCD (mean $0.270 \pm$ 0.002 ) are significantly lower than the corresponding values for PMN from the control group (mean $0.288 \pm 0.003$ ).

When resting PMN from the PCD and control children were stimulated with FMLP, a statistically significant $(p<0.05)$ increase in $r_{\mathrm{s}}$ value was observed in both groups.

Figures 1 and 2 show TMA-DPH fluorescence lifetime distribution in PMN from the control and PCD groups. Data analysis revealed the presence of two distinct components of lifetime distribution in the samples. In the control group, a long component with a central lifetime value of $6.5 \mathrm{~ns}$ (fractional intensity of 0.8 ) and a short component with a central lifetime value of $0.8 \mathrm{~ns}$ (fractional intensity of 0.2 ) were observed. The width of the distribution was $0.30 \mathrm{~ns}$ for the long component and $0.6 \mathrm{~ns}$ for the short one. In PMN from the PCD group, the long component has a central lifetime value of $6.3 \mathrm{~ns}$ (fractional intensity of 0.8 ) and the short component has a central lifetime

Table 1. Mean $\pm S D$ of $r_{s}$ of $T M A-D P H$ at $37^{\circ} \mathrm{C}$ in $P M N$ from control and PCD groups in basal conditions and after stimulation with FMLP

\begin{tabular}{lcc}
\hline & \multicolumn{2}{c}{$\mathrm{r}_{\mathrm{s}}$} \\
\cline { 2 - 3 } & $\begin{array}{c}\text { Controls } \\
(n=17)\end{array}$ & $\begin{array}{c}\text { PCD } \\
(n=7)\end{array}$ \\
\hline Unstimulated PMN & $0.288 \pm 0.003$ & $0.270 \pm 0.002^{*}$ \\
Stimulated PMN & $0.360 \pm 0.004^{*}$ & $0.300 \pm 0.001 \dagger \neq$ \\
\hline
\end{tabular}

$* p<0.05$ compared with unstimulated PMN from the control group. $\dagger p<0.05$ compared with unstimulated PMN from the PCD group.

$\ddagger p<0.05$ compared with stimulated PMN from the control group.

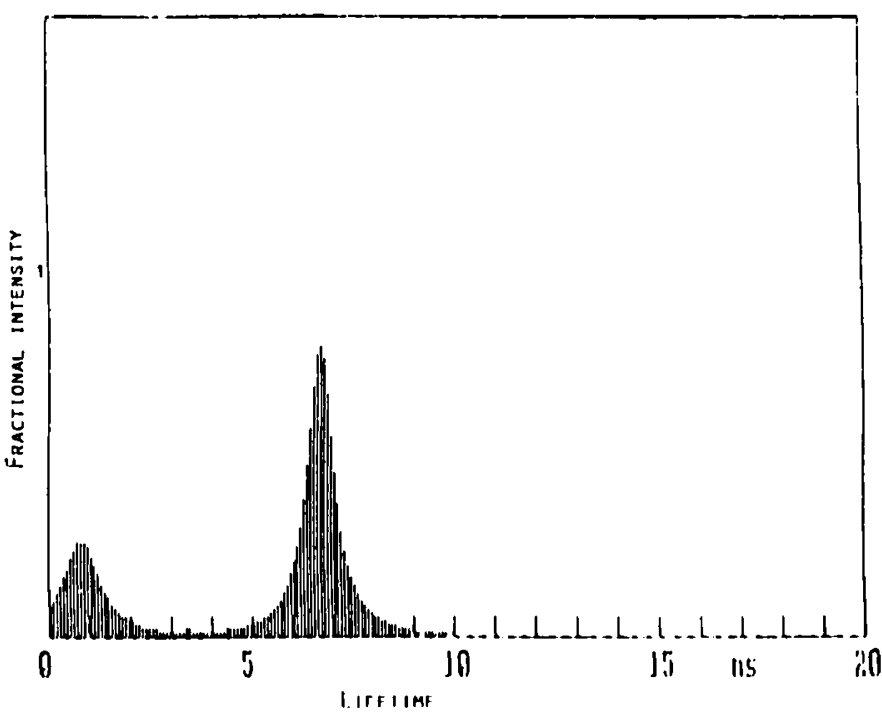

Fig. 1. TMA-DPH lifetime distribution in PMN from the control group. The distribution width of the long component has a value of 0.30 ns and is centered at $6.5 \mathrm{~ns}$. 


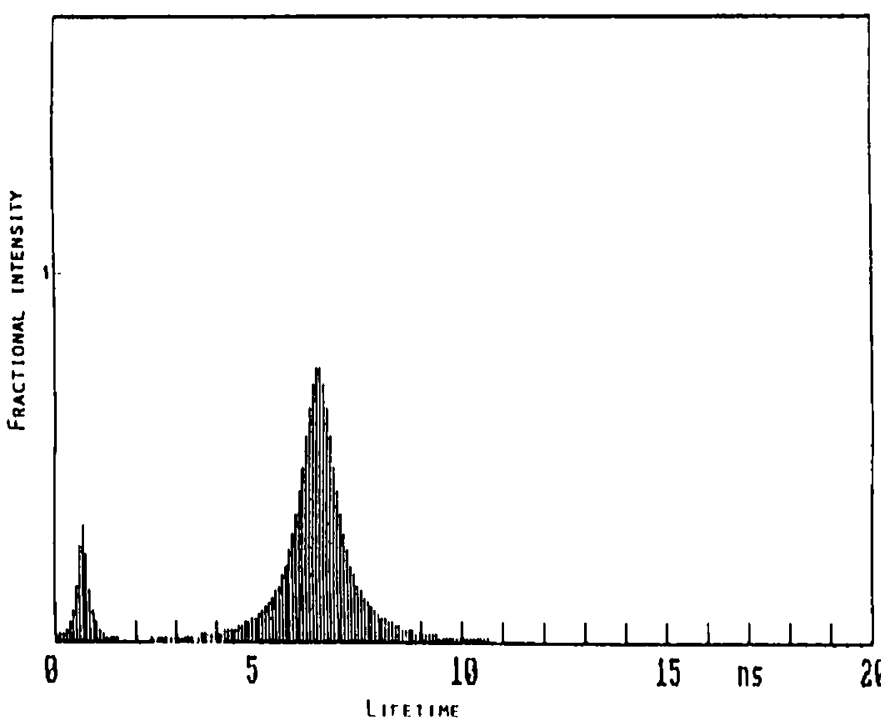

Fig. 2. TMA-DPH lifetime distribution in PMN from children with PCD. The distribution width of the long component has a value of 0.5 ns and is centered at $6.3 \mathrm{~ns}$.

value of $0.8 \mathrm{~ns}$ (fractional intensity of 0.2 ). The distribution widths were $0.5 \mathrm{~ns}$ for the long component and $0.05 \mathrm{~ns}$ for the short component.

\section{DISCUSSION}

Biologic membranes are a complex mixture of different types of lipid and protein molecules. Different modes of organization of the compositionally and functionally differentiated domains correspond to different functional states of the membrane (23). Chemical and physical events that take place within the membrane allow the cells to carry out their specific functions (23). Fluorescence spectroscopy is a useful tool in the study of the structure and function of biologic membranes because its high sensitivity, low degree of membrane perturbation, and favorable time scale allow one to observe a wide range of molecular processes (24). TMA-DPH has been used intensively for studying membrane structure and fluidity because of its advantageous structural and photophysical properties $(25,26)$. Because of its hydrophobic structure, TMA-DPH incorporates into the membrane but remains at the lipid-water interface region because of its cationic residue (26). TMA-DPH $r_{s}$ reflects the packing of membrane lipid fatty acid chains and can be related to the order parameter $S$, if certain precautions are taken $(27,28)$. Lipid fluidity may be defined as the reciprocal of the lipid structural order parameter S (27), and thus a decrease of TMA-DPH $r_{\mathbf{s}}$ value corresponds to an increase in membrane fluidity. In our study, we have found that $r_{s}$ of TMA-DPH in resting PMN from the PCD group is significantly lower than that in PMN from the control children. This indicates an increase in membrane fluidity that reflects a decrease in lipid ordering in the exterior part of PMN plasma membrane from patients with PCD. After stimulation of PMN from both groups with FMLP, significant differences in $r_{s}$ values were observed between the two groups.

The TMA-DPH fluorescence lifetime value is dependent on the dielectric constant of the medium in which the probe is embedded (29). Therefore, the width of the lifetime distribution can be related to the different physicochemical properties of the environment surrounding the probe. The distribution analysis, although based on phenomenologic ground, offers a good description of membrane heterogeneity $(20,21,30)$. In our distribution analysis, it was necessary to include a second component at a shorter lifetime with a very low fractional intensity. The origin of this second component is still debated; for diphenylhexatriene, this component has been referred to as a photochemical derivative of the probe (31) or, alternatively, it can represent a fraction of probe molecules localized in a very polar environment (32). In any case, the relative fractional intensity of the short component did not differ significantly between the two groups. Our data for TMA-DPH lifetime distribution show that PMN from children with PCD have a broad distribution width of the long component with respect to the control PMN. No significant differences were observed in the central lifetime value between the two groups. These results indicate an increase in membrane heterogeneity of PMN in children with PCD.

The plasma membrane is a complex structure exhibiting specific and dynamic interactions between cytoskeletal proteins and membrane lipids that determine the mobility and distribution of these constituents (33).

Functional studies of PMN motility implicating a requirement for an integrated cytoplasmic microtubule-membrane interaction have been recently reported (33-35). Decreased chemotaxis associated with alterations in membrane fluidity was reported in PMN treated with microtubule disrupting agents such as lead or 2,5 -hexanedione $(15,36)$ in PMN from subjects with ChédiakHigashi syndrome $(34)$ or from healthy newborn infants $(37,38)$. Cytoskeletal architecture might directly affect the local composition of the plasma membrane $(34,35)$. Cytoskeleton-related changes in the lipid composition of the membrane or in membrane surface tension might afford regions of minimal free energy to certain classes of molecules such as chemotactic receptors (39).

The finding of an impaired chemotactic activity of PMN from the PCD group is consistent with previous studies (1-5) that have attributed the reduced motility to alterations in the cytoskeleton. Because the cytoskeleton can influence the distribution and dynamics of membrane components, we could speculate that alterations in the cytoskeleton may result in the above-described changes of membrane fluidity and heterogeneity in PMN from subjects with PCD.

Experimental evidence indicates that each integral membrane protein interacts with its neighboring "boundary" lipids in a specific manner (40). Alterations in the composition of this lipid boundary may lead to changes in enzyme activity as well as in ligand specificity and affinity (41).

Recently, Wolach et al. (38), using diphenylhexatriene as a fluorescent probe, have indicated that increased fluidity in neonatal PMN contribute to their deficiency in chemotactic ability, demonstrating an improvement of chemotactic capacity after membrane rigidification (38). At the moment, we cannot exclude the existence of a primary defect in membrane composition and organization. This hypothesis may be supported by the observation reported by Wolburg et al. (4) of a reduced number of intramembranous particles in PMN from PCD patients.

In conclusion, we believe that the observed alterations in the physicochemical structure of plasma membranes may contribute to the impairment of motility of PMN from children with PCD.

\section{REFERENCES}

1. Afzelius BA, Ewetz L, Palmblad J, Uden AM, Venizelos N 1980 Structure and function of neutrophil leukocytes from patients with the immotile-cilia syndrome. Acta Med Scand 208:145-154

2. Englander LL, Malech HL 1981 Abnormal movement of polymorphonuclear neutrophils in the immotile cilia syndrome. Exp Cell Res 135:468-472

3. Valerius NH, Knudsen BB, Pedersen M 1983 Defective neutrophil motility in patients with primary ciliary dyskinesia. Eur J Clin Invest 13:489-494

4. Wolburg H, Dopfer R, Schieferstein G, Theil E 1984 Immotile cilia syndrome: reduced chemotaxis and reduced number of intramembranous particles in granulocytes. Klin Wochenschr 62:1044-1046

5. Canciani M, Barlocco EG, Cazzola GA, Mastella G, de Santi MM, Gardi C, Lungarella $G 1988$ The association of supernumerary microtubules and immotile cilia syndrome and defective neutrophil chemotaxis. Acta Paediatr Scand 77:606-607

6. Lenaz G 1979 The role of lipids in the structure and function of membranes. In: Roodyn DB (ed) Subcellular Biochemistry, Vol 6. Plenum Press, New York, pp 233-343

7. Shinitzky M 1984 Membrane fluidity and cellular functions. In: Shinitzky M (ed) Physiology of Membrane Fluidity. CRC Press, Boca Raton, FL, pp 1- 
8. Lenaz G, Curatola G, Fiorini R, Parenti-Castelli G 1983 Membrane fluidity and its role in the regulation of cellular processes. Biol Cancer 2:25-34

9. Kantar A, Giorgi PL, Curatola G, Fiorini R 1992 Alterations in erythrocyte membrane fluidity in children with trisomy 21 : a fluorescence study. Biol Cell 75:135-138

10. Giorgi PL, Oggiano N, Braga PC, Catassi C, Gabrielli O, Coppa GV, Kantar A 1992 Cilia in children with recurrent upper respiratory tract infections: ultrastructural observations. Pediatr Pulmonol 14:201-205

11. Giorgi PL, Oggiano N, Kantar A, Fabbrizi E, Piatti G, Braga PC 1991 Analysis of respiratory cilia motion and beat frequency counting in screening for primary ciliary dyskinesia in pediatric age. Riv Ital Pediatr 17:707-711

12. Giorgi PL, Oggiano N, Biraghi M, Kantar A 1991 Effect of high doses of inhaled flunisolide on hypothalamic-pituitary-adrenal axis function in children with asthma. Curr Ther Res 49:778-783

13. Kantar A, Wilkins G, Swoboda B, Littarnu GP, Bertoli E, Catassi C, Coppa G, Giorgi PL 1990 Alterations of the respiratory burst of polymorphonuclear leukocytes from diabetic children. Acta Paediatr Scand 79:535-54

14. Kantar A, Oggiano N, Romagnoni GG, Giorgi PL 1991 Effect of oral admin istration of bacterial extracts on the bactericidal capacity of polymorphonuclear leucocytes in children with recurrent respiratory infections. J Int Med Res 19:451-456

15. Governa M, Valentino M, Visona I, Rocco M 1986 Impairment of human polymorphonuclear leukocyte chemotaxis by 2,5 -hexanedione. Cell Biol Toxicol 2:33-39

16. Metcalf JA, Gallin JI, Nauseef WM, Root RK 1986 Laboratory Manual of Neutrophil Function. Raven Press, New York, pp 22-23

17. Kantar A, Giorgi PL, Curatola G, Fiorini R 1991 Alterations in membrane fluidity of diabetic polymorphonuclear leukocytes. Biochem Med Metab Biol 46:422-426

18. Fiorini R, Curatola G, Bertoli E, Giorgi PL, Kantar A 1990 Changes of fluorescence anisotropy in plasma membrane of human polymorphonuclear leukocytes during the respiratory burst phenomenon. FEBS Lett 273:122126

19. Fiorini R, Valentino M, Gratton E, Bertoli E, Curatola G 1987 Erythrocyte membrane heterogeneity studied using 1,6-diphenyl-1,3,5-hexatriene fluorescence lifetime distribution. Biochem Biophys Res Commun 147:460-466

20. Kantar A, Giorgi PL, Curatola G, Fiorini R 1992 Changes in plasma membrane microheterogeneity of polymorphonuclear leukocytes during the activation of the respiratory burst. In: Jesaitis AJ, Dratz EA (eds) Molecular Basis of Oxidative Damage by Leukocytes. CRC Press, Boca Raton, FL, pp 243-246

21. Kantar A, Giorgi PL, Curatola G, Fiorini R 1991 Effect of PAF on erythrocyte membrane heterogeneity: a fluorescence study. Agents Actions 32:347-350

22. Fiorini R, Valentino M, Wang S, Glaser M, Gratton E 1987 Fluorescence lifetime distribution of 1,6-diphenyl-1,3,5-hexatriene in phospholipid vesicles. Biochemistry 26:3864-3870

23. Kinnunen PKJ 1991 On the principles of functional ordering in biological membranes. Chem Phys Lipids 57:375-399

24. Van Der Meer BW 1988 Biomembranes structure and dynamics viewed by fluorescence. In: Hilderson HJ (ed) Fluorescence Studies on Biological Membranes, Vol 13. Plenum Press, New York, pp 1-53

25. Lentz BR 1989 Membrane "fluidity" as detected by diphenylhexatriene probes. Chem Phys Lipids 50:171-190

26. Kuhry JG, Fonteneau P, Duportail G, Maechling C, Laustriat G 1983 TMA DPH: a suitable fluorescence polarization probe for specific plasma membrane fluidity studies in intact cells. Cell Biophys 5:129-140

27. Van Blitterswijk WJ, Van Hoeven RP, Van Der Meer BW 1981 Lipid structural order parameters (reciprocal of fluidity) in biomembranes derived from steady-state fluorescence polarization measurements. Biochim Biophys Acta 6teady-state fluor

28. Pottel H, Van Der Meer W, Herreman W 1983 Correlation between the order parameters and the steady state fluorescence anisotropy of 1,6-diphenyl1,3,5-hexatriene and an evaluation of membrane fluidity. Biochim Biophys Acta 730:181-186

29. Zannoni C, Arcioni A, Cavatorta $P 1983$ Fluorescence depolarization in liquid crystals and membrane bilayers. Chem Phys Lipids 32:179-250

30. Fiorini R, Valentino M, Glaser M, Gratton E, Curatola G 1988 Fluorescence lifetime distributions of 1,6-diphenyl-1,3,5-hexatriene reveal the effect of cholesterol on the microheterogeneity of erythrocyte membrane. Biochim Biophys Acta 939:485-492

31. Parasassi T, Conti F, Glaser M, Gratton E 1984 Detection of phospholipid phase separation: a multifrequency phase fluorimetry study of 1,6-diphenyl1,3,5-hexatriene fluorescence. J Biol Chem 259:14011-14017

32. Klausner RD, Kleinfeld AM, Hoover RL, Karnovsky MJ 1980 Lipid domains in membranes: evidence derived from structural perturbations induced by free fatty acids and lifetime, heterogeneity analysis. J Biol Chem 255:12861295

33. Jesaitis AJ, Allen RA 1988 Activation of the neutrophil respiratory burst by chemoattractants: regulation of the $\mathrm{N}$-formyl peptide receptor in the plasma membrane. J Bioenergy Biomembr 20:679-707

34. Baehner RL, Boxer LA 1979 Disorders of polymorphonuclear leukocyte function related to alterations in the integrated reactions of cytoplasmic constituents with plasma membrane. Semin Hematol 16:148-162

35. Oliver JM, Berlin RD 1983 Surface and cytoskeletal events regulating leukocyte membrane topography. Semin Hematol 20:282-304

36. Governa $M$, Valentino $M$, Visona I 1987 In vitro impairment of human granulocyte function by lead. Arch Toxicol 59:421-425

37. Harris MC, Shalit M, Southwick FS 1993 Diminished actin polymerization by neutrophils from newborn infants. Pediatr Res 33:27-31

38. Wolach B, Ben Dor M, Chomsky O, Gavrieli R, Shinitzky M 1992 Improved chemotactic ability of neonatal polymorphonuclear cells induced by mild membrane rigidification. J Leukoc Biol 51:324-328

39. Walter RJ, Berlin RD, Oliver JM 1980 Asymmetric Fc receptor distribution of human PMN oriented in a chemotactic gradient. Nature 286:724-725

40. Watts A 1989 Membrane structure and dynamics. Curr Opin Cell Biol 1:691700

41. Conforti G, Zanetti A, Pasquali-Ronchetti I, Quaglino D, Neyroz P, Dejana E 1990 Modulation of vitronectin receptor binding by membrane lipid composition. J Biol Chem 265:4011-4019 